ISSN 1112-9867

Available online at

http://www.jfas.info

\title{
EFFECT OF EARTH DISCONTINUED TO THE ELECTRICAL FIELD DISTRIBUTION IN ROD-PLANE AIR GAPS UNDER LIGHTNING IMPULSE
}

\author{
A. Khechekhouche and D. Ben Attous \\ Department of Electrical Engineering Biskra, University of Biskra, Algeria,BP145, 07000 Biskra, \\ Algeria
}

Received: 18 June 2016 / Accepted: 30 August 2016 / Published online: 01 September 2016

\begin{abstract}
This paper, will present some results of investigations carried out on electric field distribution on the surface of a discontinuous plane of short rod-plane air gap arrangement. For this purpose, we have used different results obtained by an experimental model and a numerical method for computation used the finite elements methods (FEM). The effect of interface on the field stress has also been studied, As a result, the interface locally reinforces the electric field, and then the electric field increases at the interface may lead to a discharge between the high voltage rode and the interface. In the vicinity of the interface, we have observed a kind of discontinuity in the evolution of the electric field intensity. This latter becomes greater than the value obtained in the case of gaps with homogeneous plane earth. The electric field distribution, obtained by means of the computational procedure, is in good agreement with the experimental results.
\end{abstract}

Keywords: discontinuous plane; the electric field; homogeneous plane; the finite elements methods (FEM).

Author Correspondence, e-mail: khechali@yahoo.fr

doi: http://dx.doi.org/10.4314/jfas.v8i3.21 


\section{INTRODUCTION}

In the field lightning protection several models had been proposed by different authors. The more studied case is the negative descending strike. In such case, when the steeped leader reaches its critical position, the final jumps occur with a probability overloading to the principal discharge (return strike) characterized by the ascending positive current flow from the earth to the storm cloud, neutralizing the negative charges situated at the basis of this latter [1-3].

In some electrode geometries, the electric fields can simply be expressed analytically in a closed form solution; in another way, the electric field problem is complex because of the sophisticated boundary conditions, including media with different permittivity and conductivities. For assessing the electric field distributions in the complex arrangements, sometimes in three dimensions, analytical methods are by no means suitable. In such cases the other approaches are in use, numerical techniques and experimental analogs. Numerical methods are particularly useful when the analytical solution is very complicated or impossible [1].

Electric field problems in high voltage technique are mostly electrostatic field problem. The calculation of electrostatic fields requires the solution of Poisson's and Laplace's equations with boundary condition satisfied. Several numerical techniques have been used in the literature for solving Laplace's and Poisson's equations for the fields between complex electrode arrangements [4-7]. Finite element method FEM is one of the most successful numerical methods for solving electrostatic field problems. FEM can be employed successfully for the computation of an electric field between electrodes in a medium where one or more dielectrics are involved. Solution of the electric field problems by FEM is based on the fact, known from variational calculus, that Laplace's equation is satisfied when the total energy functional is minimal [8-10].

Several models have been proposed for the calculation of the electric field and charge density distribution using the finite element techniques [11] , the calculation of the electrical quantities is performed using a finite element method. The calculated of electric field distribution are compared to measured ones carried out on laboratory model of rod-plane air gap arrangement. Therefore, the aim of the present work is to validate a numerical model and to show that modeling of electric field under lightning impulse voltage can successfully be performed using finite element techniques [12-15]. 


\section{EXPERIMENTAL SET UP}

The used arrangement with homogeneous system is made up of a square metallic sheet (Fig.1). The high voltage (HV) rod is made up of steel with $4.8 \mathrm{~mm}$ diameter, and hemispheric end shape. The applied voltage is given by a $(-1.2 / 50 \mu \mathrm{s})$ lightning impulse generator of $600 \mathrm{kV}, 4 \mathrm{~kJ}$. The position of the rod is determined by its height $\mathrm{h}$ compared to the earth. The studied earth called "discontinuous earth" is made up of a square metallic sheet with $10 \mathrm{~mm}$ thickness and having two different levels of respective surface $1.2 \times 1.2 \mathrm{~m}^{2}$ (Fig.1). The position of the rod is determined by its height (h) compared to the earth and the distance (D) between its axis and the interface. This distance is considered positive when the rod is located above the high and negative part in the other case.

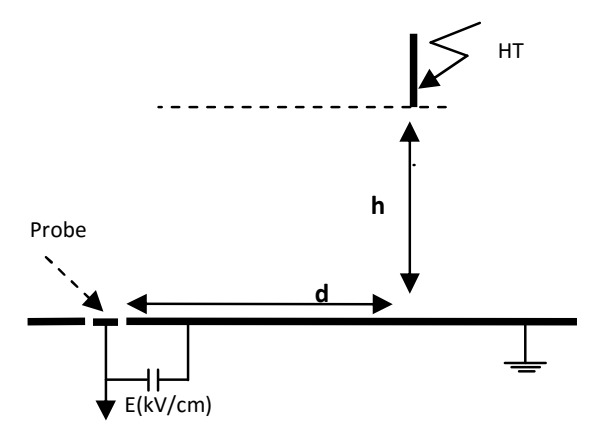

(a) homogeneous system

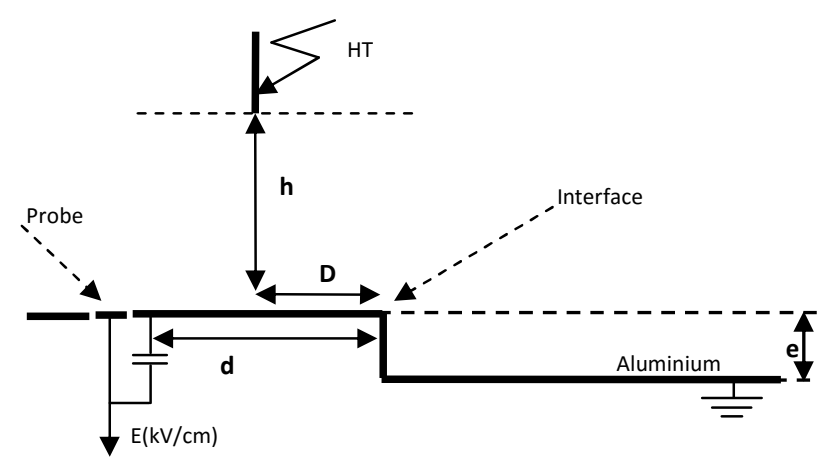

(b) discontinuous system

Fig.1. Experimental arrangement for electric field measurement

The distances $\mathrm{h}$ are the same one as in homogeneous system, but the distances $\mathrm{d}$ and $\mathrm{D}$ are selected so that the tests are carried out for $\mathrm{d} / \mathrm{h}$ ratio and respectively identical $\mathrm{D} / \mathrm{h}$ for various values of $\mathrm{h}$. The applied voltage level to use is $0.5 \mathrm{U}_{0 \%}$ of the rod-plane corresponding system, at each selected distance h. For a given height h, we as well apply the same applied voltage level for the rod located above the high part as above the low part of the discontinuous plane [1].

Thus it is the same level of applied voltage $\mathrm{U}_{0 \%}$ which is taken into account for a given height $\mathrm{h}$ and the corresponding height $\mathrm{h}+\mathrm{e}$ when the rod is located above the low part of the discontinuous plan. We are interesting to the $\mathrm{U}_{0 \%}$ voltage of disruptive discharge in order to determine the voltage level of selected test equal to $0.5 \mathrm{U}_{0 \%}$. This applied voltage level allow to avoid the disruptive discharges on the level of the probe because of its sensitivity to the high currents. We use these measurements results in the model mathematic. 


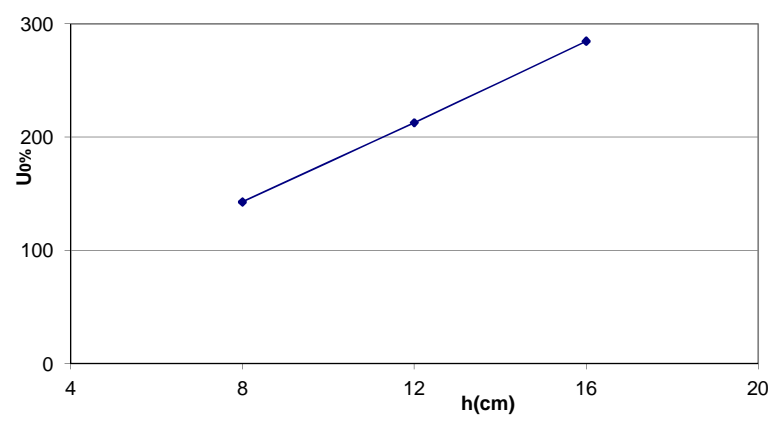

Fig.2. $\mathrm{U}_{0 \%}$ voltage with regard the air interval $h$.

We consider $\mathrm{U}_{0 \%}$ as being the voltage having a probability of discharge equal to $0.1 \%$ on the Gausso-arithmetic scale. According to measurements, we note that the applied voltage $\mathrm{U}_{0 \%}$ grows linearly with the increase length of the interval of air h (Fig.2).

The obtained values of $\mathrm{U}_{0 \%}$ for 8,12 and $16 \mathrm{~cm}$ are also taken as voltage level of tests in the case of the measurements of field for the positions of the stem to the top of the low part of the discontinuous plane; the corresponding distances as far as field will be respectively 18, 22 and 26 $\mathrm{cm}$.

\section{PROBLEM DEFINITION AND FORMULATION}

In this study, the electric field distribution in a rod-plane gap with a discontinuous plane is numerically analyzed by using finite element method (FEM). For applications of ac having extra low frequency as $50 / 60 \mathrm{~Hz}$ or dc voltages, problems may be considered an electrostatic field problem and therefore the electric and magnetic field components may be considered independently of each other, and calculations made on the basis of static field concepts. In the case of electrostatic (and also quasi-static) fields since other electromagnetic fields Maxwell's equations are the governing equations [8-10]. In electrostatics, Maxwell's equations and constitutive equation reduce to the following form

$$
\begin{array}{ll}
\ddot{\vec{B}} \cdot \vec{E}=\rho / \varepsilon_{0} & \text { (Gauss's law) } \\
\vec{\nabla} \cdot \vec{J}=0 & \text { (Current continuity condition) } \\
\vec{J}=\rho . \mu . \overrightarrow{\vec{l}} \quad \text { (Equation of current density) }
\end{array}
$$




$$
\vec{F}=-i \overrightarrow{7} \varphi \text { (Equation relating the electric field to the potential) }
$$

Where $J$ is the current density vector, $\rho$ the ionic charge density, $\varepsilon_{0}$ is permittivity of free space, $\mu$ the ionic mobility (assumed constant in this work), $E$ the electric field, and $\varphi$ the electric potential. By sabstituting (4) into (1), we can obtain the familiar Poisson's equation:

$$
\overrightarrow{7} \cdot \overrightarrow{7} \varphi=\rho / \varepsilon
$$

and substituting (3) into (2), expanding the divergence, taking into account of (1) and (4) provides an equation that governs the evolution of charge density in inter-electrode space:

$$
\mid \overrightarrow{7} \rho \cdot \overrightarrow{7} \varphi-\frac{\rho^{2}}{\varepsilon_{0}}=0
$$

In this study, solution of the problem is obtained from solution of Laplace's equation in cylindrical coordinates. In circular-cylindrical coordinates $(r, \theta, z)$, three-dimensional expression of Laplace's equation is

$$
\frac{\partial^{2} V(r, \theta, Z)}{\partial r^{2}}+\frac{1}{r} \frac{\partial V(r, \theta, Z)}{\partial r}+\frac{1}{r^{2}} \frac{\partial^{2} V(r, \theta, Z)}{\partial \theta^{2}}+\frac{\partial^{2} V(r, Z)}{\partial Z^{2}}=0
$$

Solution of equation (7) depends on boundary conditions of the problem. When the problem is solved by FEM, all boundary conditions of the problem must be known. The rod-plane gap is an open boundary problem. It means the field may extend to infinity.

\section{RESULTS AND DISCUSSION}

\subsection{Electric Field with Homogeneous Earth}

In order to obtain a reference for the comparison between the results of experimental and numerical computation with discontinuous system, we initially determine the distribution of the field in the case of the homogeneous system, according to the height $\mathrm{h}$ of the stem under high voltage in the case of the selected voltage level $0.5 \mathrm{U}_{0 \%}$ (Fig.1).

With the applied voltage level $0.5 \mathrm{U} 0 \%$, we notice that, the intensity of the electric field tends towards a constant value, as the probe moves away from the rod under voltage starting from a relative position $\mathrm{d} / \mathrm{h}$ higher or equalizes to 2 . When the probe approaches the axis of the rod for $\mathrm{d} / \mathrm{h}$ lower than 2 , the intensity of the field increases slightly and takes a maximum value for $\mathrm{d} / \mathrm{h} \leq 1$, (Fig.3). 
For the various positions of the probe $(\mathrm{d} / \mathrm{h})$ for the applied voltage level, we notice that the length of the air interval (h) has a great influence on the value of the field on the earth. Indeed, we notice that the intensity of the field increases with the height $h$. This increase would occur the applied voltage level at the rod, corresponding to $0,5 \mathrm{U}_{0 \%}$ of the interval. For the intervals $\mathrm{h}=18 \mathrm{~cm}, 22 \mathrm{~cm}$ and $26 \mathrm{~cm}$, one same applied voltage $\mathrm{U}_{0 \%}$ as the intervals $\mathrm{h}=8 \mathrm{~cm}, 12 \mathrm{~cm}$ and $16 \mathrm{~cm}$ respectively, which explains the reduction in the field for these great intervals. The increase in the field on the earth could be explained by the presence of the field creates by the space charge in the vicinity of the rod when the applied voltage level increases (Fig.3).

The results obtained from FEM analysis show that the equipotential lines are condensed around the rod and field distribution in the gap is non-uniform. The field at the rod surface reaches its maximum value at the point facing the grounded plane.
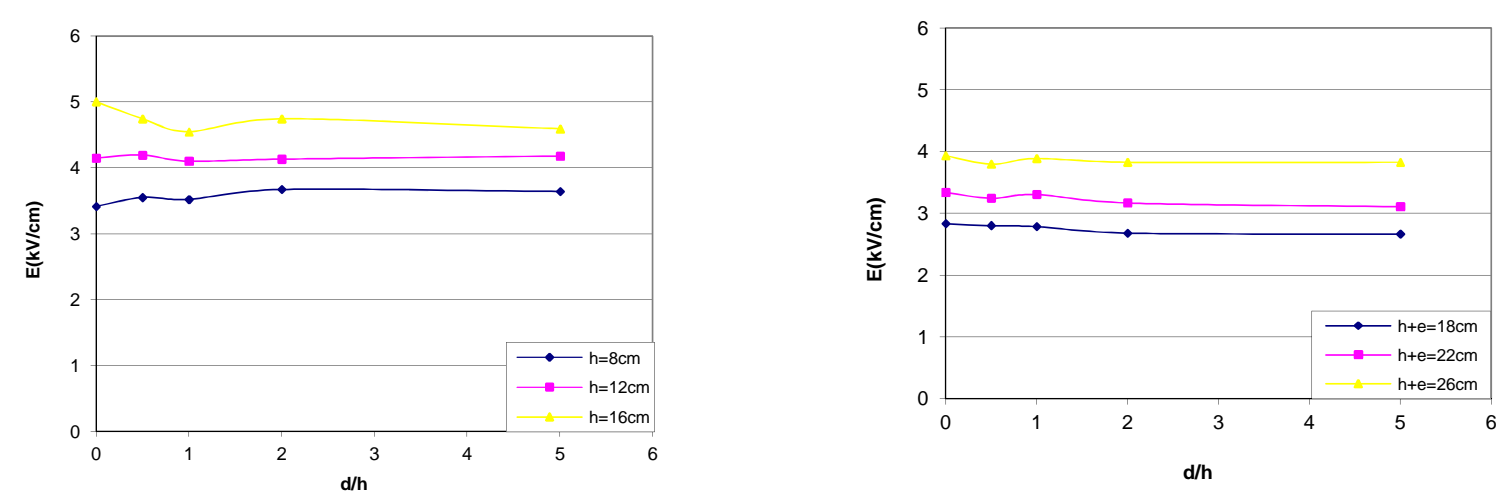

Fig.3. Electric field measured on the earth according to the position $\mathrm{d} / \mathrm{h}$ of the probe (Voltage level $\mathrm{U}=0.5 \mathrm{U} 0 \%$ )

\subsection{Electric Field with Discontinuous Earth}

After the determination of the voltage $\mathrm{U}_{0 \%}$ for the various heights $\mathrm{h}$ and the measurement of the electric field of the homogeneous system conducting, we proceed to the study of the various discontinuous earth configurations (Fig.1). We distinguish a-two-system requirements rod-plan in the case of the discontinuous earth which were defined according to positions of the rod compared to the two parts of the discontinuous earth.

\subsubsection{Case of the Rod at the Top of the High Part of Discontinuous Earth}

For the applied voltage level $0.5 \mathrm{U}_{0 \%}$ and for the positions of the probe far from the interface $(d / h$ $>2$ ), we notice that the intensity of the field measured on the high part of the discontinuous earth 
is practically equal to that corresponding to the case of the system with homogeneous earth of the same length ( Fig.4). Near the interface $(d / h<2)$, the results obtained show a kind of discontinuity in the evolution of the electric field. This discontinuity is due to the role of the interface which would have the same effect as a point, transforming the system rod-plan into system rod-rod. This fact explains the abrupt increase in the field above the interface and the reduction in the rigidity of the interval rod-interface and the phenomena of discharge observed in preceding research tasks [1]. Indeed, even for relatively large D/h distances, electric discharges can occur between the rod and the interface and not between the rod and the plan according to the path the shortest correspondent with the height h (Fig.2).

We notice that the air interval rod-interface constitutes preferable path for the lines of field. Indeed, the system rod-interface which behaves as a system point-point is less rigid than the system point-plan. This justifies, the reason why, we sometimes obtain disruptive discharges on the interface, for the low values of the $\mathrm{D} / \mathrm{h}$ ratio.

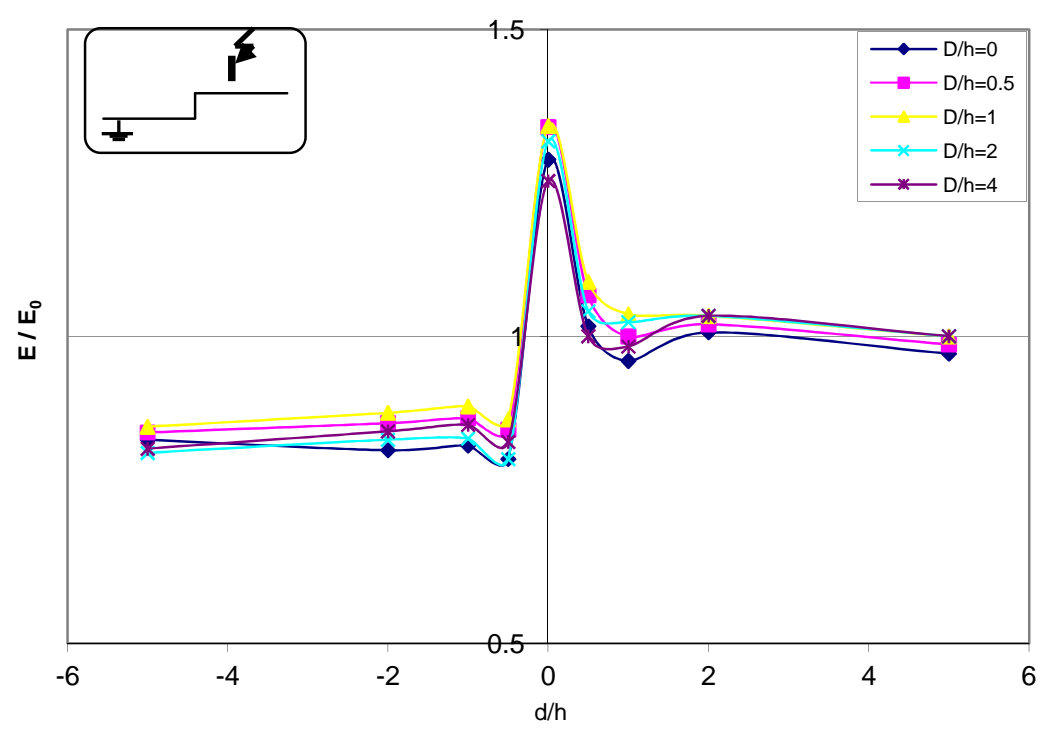

Fig. 4. shows the variation of the electrical field distribution for various position obtained by experimental measurements, with $\mathrm{E}_{0}=4.6 \mathrm{kV} / \mathrm{cm}, \mathrm{h}=16 \mathrm{~cm}$. [1]

In this study, numerical computations are performed by using FEM technique. Electric field of the rod-plane gap (Fig 5) is obtained by applying the initial and boundary conditions. Initial condition applies when there is no charge in the gap before the application of the voltage. We 
assumed that the applied voltage is 1 Volt across $16 \mathrm{~cm}$ gap distance in air at atmospheric pressure. Fig 5 shows the computed electric field distribution on the discontinuous plane when the rod is located on the high part of the discontinuous plane.
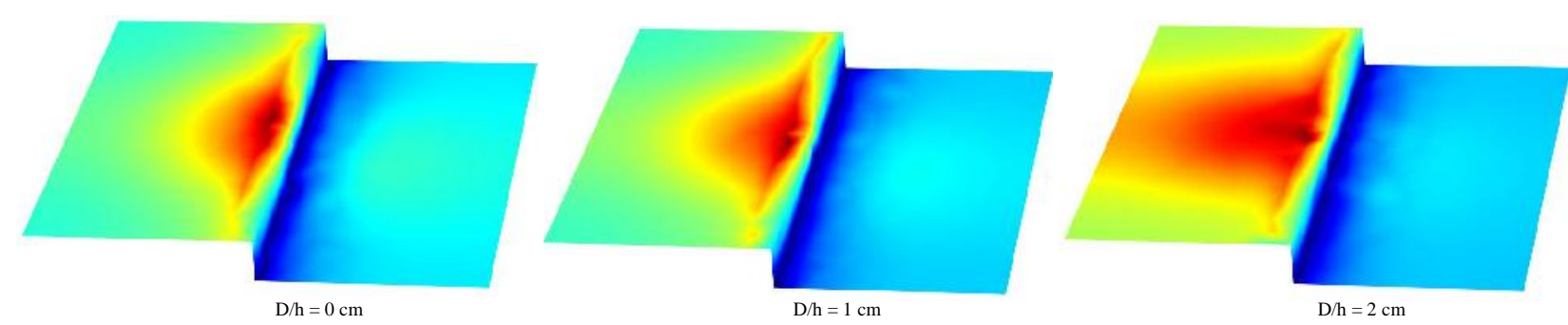

Fig.5. The electric field distributions on the discontinuous plane, Obtained by FEM.

D distance Rod-Interface, $\mathrm{h}=16 \mathrm{~cm}$

The most important factor influencing the occurrence and characteristics of electrical discharges on a gap is the electric field distribution on the plane. An analysis of discharge performance requires information on the variation of electric field around the interface of the discontinuous earth and especially maximum electric field on the interface.

If maximum field intensity is greater than electric strength of dielectric medium of the gap, an electrical discharge such as partial discharge, corona or breakdown begins from the maximum field point in the dielectric. Therefore, accurate determination of electric field distribution and maximum field is an important pre-requisite to the evaluation of the discharge performance of HV apparatus and components.

Electric field calculations are carried out for different distances between a rod and interface (Fig 5). At the end of each computation maximum field value was easily read on surface plot created by the FEM program for electric field distribution.

The results obtained from FEM analysis show that the equipotential lines are condensed around the interface and field distribution in the gap is non-uniform. The field at the interface surface reaches its maximum value. This method was tested with several experimental measurements and it was observed that the magnitudes of the field intensity calculated are lower than the measurements. This gives a conservative value that can be used to design protection distances. 


\subsubsection{Case of the Rod at the Top of the Low Part of Discontinuous Earth}

With regard to the field on the low part of the discontinuous earth, the results show that the field intensity is lower than that of the high part. That is due to the increase in the distance rod-probe. In the vicinity of the interface, the field takes the lowest value on the plan (Fig.6).

In this case where the rod is located above the high part of the plan (Fig 6), we notice that report $(E / E O)$ is close to the unit, for the position of the probe far from the interface $(d / h>1)$.

On the level of the interface, where the system rod-interface behaves as a system rod-rod which is less rigid than the system rod-plan, the relationship between the two systems takes the value maximum $(E / E O=1.25)$ which corresponds to the value where there is a great probability of having disruptive discharges direct on the interface (Fig. 6).

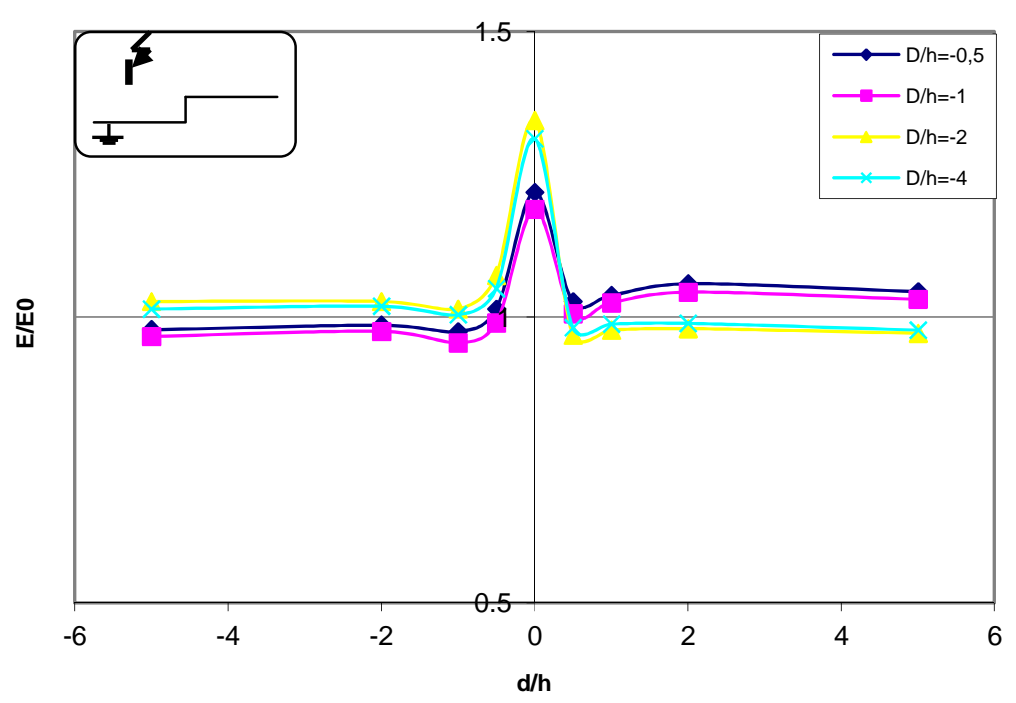

Fig.6. shows the variation of the electrical field distribution for various position obtained by experimental measurements, with $\mathrm{E}_{0}=3.82 \mathrm{kV} / \mathrm{cm}, \mathrm{h}=16 \mathrm{~cm}$.[1]

Here, the rod is inserted in the low part of the discontinuous earth and field distributions for different cases of positions are examined. In the calculations the rod was horizontally moved. Figure 7, shown an example for the obtained electric field distribution on the discontinuous plane. 


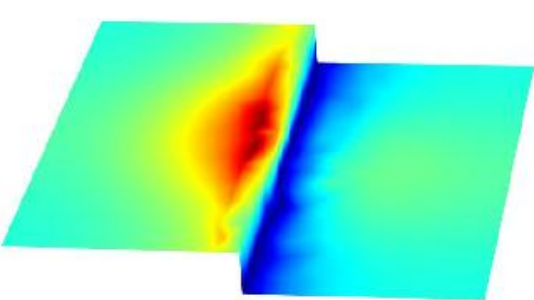

$\mathrm{D} / \mathrm{h}=0.5$

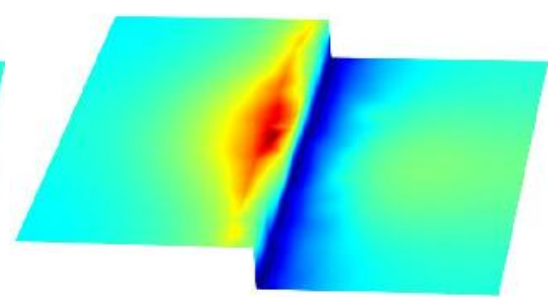

$\mathrm{D} / \mathrm{h}=1$

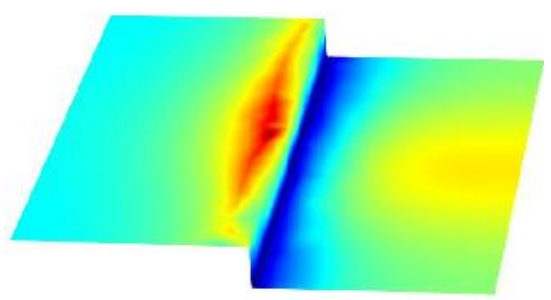

$\mathrm{D} / \mathrm{h}=2$

Fig.7. The electric field distributions on the discontinuous plane, obtained by FEM.

D distance Rod-Interface , $\mathrm{h}=16 \mathrm{~cm}$

In this figure, the presence of the rod on the low part, changes the electric field distribution according to the case of field distribution on the high part. Maximum electric field on the interface quickly increases with the decreases of the distance (D) as shown in Fig 7. Small distances are critical point of view obtaining higher maximum field. From the results of the simulation it is possible to conclude that the simulations can reproduce the experiment with very good agreement.

\section{CONCLUSION}

Our work is directed towards the determination of the distribution of the field on a discontinuous earth, in order to confirm the influence of the interface on the electric field distribution. The study developed in our research highlighted the performances of the probe at capacity distributed for measuring the electric field intensity on the earth.

Using this method of measurement (probe with distributed capacity), we determined the distribution of the field on the earth, as well in the case of a homogeneous and discontinuous plan with an interface of constant depth (e).

The test results obtained lead us to make the following observations:

The intensity of the field measured or simulated on the level of the interface, is much more significant than that in other points of the earth. This is due to the fact that the interface locally reinforces the electric field, which causes the rise in this latter, and the appearance sometimes of discharges on the interface in certain cases $(\mathrm{d} / \mathrm{h}=0$ and $\mathrm{D} / \mathrm{h}=0)$, in spite of the choice of voltage level relatively low tests compared to the voltage level U0\% corresponding to $0.1 \%$ of disruptive discharge in the case of the homogeneous earth. 
In the vicinity of the interface, we observe a kind of discontinuity in the evolution of the intensity of the electric field, this one, while decreasing, becomes lower than the value obtained in the case of the good conducting homogeneous earth of the same length.

The results of the simulations have shown that the FEM can reproduce the experimental results obtained for the discontinuous plan configuration with good accuracy. The maximum electric field intensity obtained on the interface.

FEM is highly valued to the calculated electric field configurations with fairly simple programs and small computing time; it has the ability to solve the electrostatic problems very quickly and with high precision.

\section{REFERENCES}

[01] Khechekhouche A. Benattous D. Mekhaldi A. Boubakeur A. Electric field measurement in roddiscontinued plane air gaps using distributed capacity probe. J Fundam App Sci. 2014, 6(1), $1-10$

[02] I. Fofana, A. Beroul and A. Boubakeur, "Influence of Insulating Barriers on Positive Long Air Gaps", The 11the High Voltage Engineering Symposium, London, Vol. 3, p. 321, 22 27 August 1999.

[03] J. F. Hoburg, J. L. Davis, “A Student-Oriented Finite Element Program for Electrostatic Potential Problems", IEEE Trans. on Education, Vol. E-26, No.4, Nov. 1983, pp. 138 - 142.

[04] Beroual A, Rakotonandrasana J, Fofana I. Predictive dynamic model of the negative lightning discharge based on similarity with long laboratory sparks. IEEE Transactions on Dielectrics and Electrical Insulation. October 2010, Vol. 17, 5, pp. 1551 - 1561.

[05] A. Boubakeur, S. A. A. Boumaza, R. Belaicha, R. Boudissa : «Influence of earth heterogeneity on negative lightning breakdown of rod-plane air-gaps », 24th ICLP, Birmingham, 1998, pp : 473-477.

[06] A. Boubakeur, J. Ferras : « Négative lightning breakdown of rod-plane air gaps with heterogeneous earth », 5ème ISH, paper 14-26, Braunschweig ,RFA, 1987.

[07] A . Rahmani, A. Boubakeur, H. Brouri; “ Model of an horizontal lightning conductor protection in the case of earth discontinuity" International Symposium on Electromagnetic Compatibility EMC EUROPE 2002, (Sorrento) Italy 2002, pp 267-270. 
[08] S. Cristina, G. Denelli, M. Feliziani, Numerical computation of corona space charge and V-I characteristic in DC electrostatic precipitators, IEEE Trans. Ind. Appl., 27, 147-153 (1991).

[09] Pei-bai Zhou, Numerical Analysis of Electromagnetic Fields, Springer-Verlag, Berlin, 1993.

[10] A. Kara, E. Onal, O. Kalenderli, K. Mardikyan, "The Effect of Insulating Barriers on AC Breakdown Voltage in Inhomogeneous Field”, IEEE MELECON 2006 Mediterranean Electrotechnical Conference, Benalmádena (Málaga), Spain, pp. 1206-1208, 16-19 May 2006.

[11] A.Rahmani, A. Khechekhouche, A. Mekhaldi, A.Boubakeur " Electrical Strength of RodDiscontinuous Plane Air Gap Under Lightning Impulse Applied Voltage Using a Distributed Capacity Probe " IEEE-CEIDP, October 14-17, 2012, Montréal CANADA, pp 379-382.

[12] K. Yamazawa and H. Yamashita, "Calculation of the Electric Field Distribution under the Point-plane Gap Configurations using the FEM”, 1997 IEEE Annual Report - Conference on Electrical Insulation and Dielectric Phenomena, Minneapolis, pp. 648-651, 19-22 October 1997.

[13] R. H. Golde ; «Lightning : T2 », Academic Press, 2ème édition, London,(1981).

[14] G. Le Roy, C. Gary, B. Hutzler, J. Lacot, C. Dubaton : «Les propriétés diélectriques de l'air et les très hautes tensions», Ed Eyrolles, Paris, 1984, pp : 92-251.

[15] T. HORVATH : «Computation of Ligthning Protection», Research Studies Press LTD, England 1991.

\section{How to cite this article:}

Khechekhouche A, Ben Attous D. effect of earth discontinued to the electrical field distribution in rod-plane air gaps under lightning impulse. J. Fundam. Appl. Sci., 2016, 8(3), 1054-1065. 